[Vicino Oriente XVI (2012), pp. 151-165]

\title{
IL SINCRETISMO DI GENITIVO E DATIVO IN PERSIANO ANTICO*
}

\author{
Maria Carmela Benvenuto - Flavia Pompeo - \\ Sapienza Università di Roma
}

Old Persian does not have separate forms for genitive and dative, as formerly distinct grammatical categories merged into the genitive. The aim of this paper is to analyse Old Persian dative-genitive syncretism.

Keywords: morfosintassi; sincretismo; genitivo; dativo; persiano antico

\section{INTRODUZIONE}

Il sincretismo dei casi, com'è noto, è un processo che si è verificato in molte lingue indoeuropee, determinando, talora già da fasi molto antiche, la riduzione del numero dei casi rispetto al sistema tradizionalmente ricostruito per il proto-indoeuropeo. Il persiano antico è tra le lingue che documentano questo fenomeno, che ha interessato in particolare $\mathrm{i}$ casi genitivo e dativo. Dall'analisi linguistica delle iscrizioni achemenidi, infatti, emerge che il sincretismo di questi due casi si è già verificato e che il dativo è scomparso senza lasciare alcun tipo di traccia.

Date tali premesse, l'analisi del materiale documentario del persiano antico solleva per lo meno due importanti questioni: (1) quale sia la natura del sincretismo che deve essere ricostruito; (2) quali siano i meccanismi che hanno provocato la perdita del caso dativo. A queste domande si propone di rispondere il presente contributo, nel quale si teorizza che il processo di sincretismo di genitivo e dativo sia stato determinato dalla parziale sovrapposizione della sfera funzionale dei due casi, sia a livello sintattico che semantico. In particolare, la sovraestensione funzionale del genitivo ai danni del dativo avrebbe avuto come esito la polisemia semantica del morfema sopravvissuto, vale a dire quello del genitivo.

Prima di procedere con l'analisi, sono opportune alcune osservazioni introduttive. Innanzi tutto, occorre considerare sotto il profilo teorico la nozione di "sincretismo". Nel presente contributo, secondo quanto teorizzato da G. Meiser ${ }^{1}$ e, in seguito, da S. Luraghi ${ }^{2}$, questo termine è usato per indicare un processo diacronico consistente nell'integrazione di due o più categorie paradigmatiche, originariamente distinte, in una singola categoria, per mezzo della fusione delle loro forme e delle loro funzioni.

In secondo luogo, come inquadramento teorico della nostra analisi adotteremo un approccio di tipo cognitivo-funzionale, secondo il quale le forme grammaticali, casi

\footnotetext{
* I risultati presentati in questo lavoro costituiscono la seconda fase di una ricerca condotta nell'ambito del PRIN 2008 Contatto tra varietà e mutamento nella diacronia linguistica del Mediterraneo nord-orientale. La prima parte della ricerca è stata già pubblicata, cfr. Pompeo - Benvenuto 2011. Il presente contributo è da intendersi così ripartito: a M.C. Benvenuto vanno attribuiti i paragrafi 3., 4. e 4.1., mentre F. Pompeo è autrice dei paragrafi 2., 2.1., 2.2. e 4.2.; i paragrafi 1. e 5. sono stati scritti congiuntamente dalla due autrici.

1 Meiser 1992, 190.

2 Luraghi 2000
} 
compresi, sono considerate provviste di un significato in tutti i loro usi e, se polisemiche, possono essere adeguatamente descritte per mezzo del modello delle categorie radiali ${ }^{3}$.

Il contributo è organizzato nel modo seguente: nel paragrafo 2. sarà presentata una breve panoramica delle funzioni sintattiche e dei ruoli semantici del cosiddetto caso "genitivo" del persiano antico; nel paragrafo 3. saranno presi in esame i dati attinenti alla ricostruzione morfologica e il suo eventuale rapporto con il sincretismo verificatosi; il paragrafo 4. sarà, invece, dedicato all'analisi delle funzioni sintattiche (4.1.) e degli aspetti cognitivi e semantici (4.2.) che possono aver determinato la scomparsa del dativo; infine, nel paragrafo 5. sono contenute le considerazioni conclusive.

\section{IL GENITIVO DEL PERSIANO ANTICO. UNA NUOVA CATEGORIA POLISEMICA}

Possiamo affermare che il genitivo del persiano antico è, sotto vari punti di vista, un caso nuovo. Infatti, l'ampia gamma di funzioni sintattiche e ruoli semantici caratteristici di questo caso sincretico sono quelli tipici, in una prospettiva comparativa interlinguistica, non solo del genitivo indoeuropeo, ma anche del dativo. A tale proposito, nella tabella 1 sono elencati i ruoli principali associati a questi casi sia in una prospettiva tipologica che storico-comparativa.

\begin{tabular}{|l|ll|}
\hline CASO & RUOLI \\
\hline Genitivo & Possessore, Partitivo, Agente, Paziente (non prototipico) & \\
\hline Dativo & $\begin{array}{l}\text { Ricevente, Destinatario, Beneficiario, Esperiente, Meta, Scopo, Paziente (non } \\
\text { prototipico) }\end{array}$ \\
\hline
\end{tabular}

Tab. 1: Ruoli principali del genitivo e del dativo in prospettiva interlinguistica.

La distribuzione dei differenti usi del genitivo (adnominale, con preposizione ecc.) in persiano antico è, invece, schematizzata nel grafico 1, nel quale è rappresentato il totale delle attestazioni, distinguendo i pronomi personali dalle restanti forme di genitivo, vale a dire nomi e altri tipi di pronomi ${ }^{6}$.

I paragrafi seguenti presentano una rassegna estremamente sintetica degli usi del genitivo del persiano antico, dedicando maggiore attenzione alle funzioni e ai ruoli che in una prospettiva comparativa interlinguistica sono tipici del dativo ${ }^{7}$.

Cfr. Luraghi 2009; cfr. § 2.2., 4.2.

4 La schematizzazione relativa alle funzioni del genitivo è basata su Brugmann 1911, 547 e segg.; Lander 2009, 590.

5 L'elenco delle funzioni del dativo è tratto prevalentemente da Brugmann 1911, 565 e segg.; Blake 2001, 142 e segg.; Næss 2009

6 Dall'analisi del corpus delle iscrizioni achemenidi, condotta avvalendoci delle edizioni di Schmitt 1991, 2000, 2009, sono risultate circa 800 attestazioni di forme di genitivo: di queste, circa 340 sono costituite da pronomi personali (190 tonici, 150 enclitici), mentre circa 460 sono nomi e, in proporzione inferiore, pronomi dimostrativi o indefiniti.

7 Per un'analisi dettagliata si rimanda a Pompeo - Benvenuto 2011 e alla bibliografia ivi citata. 


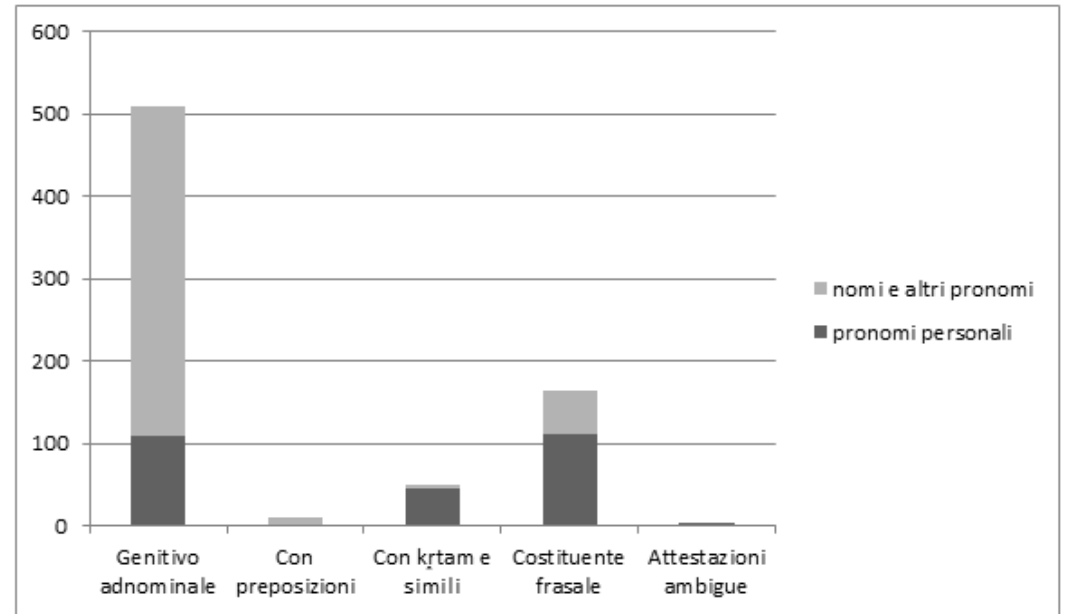

Grafico 1: Attestazioni delle forme di genitivo in persiano antico.

\subsection{Il genitivo adnominale}

Quando il genitivo del persiano antico è modificatore di un nome, le sue funzioni e i suoi ruoli sono quelli tipici di questo caso in prospettiva interlinguistica (genitivo possessivo, partitivo, soggettivo e oggettivo).

Il grafico 2 rappresenta la totalità degli usi adnominali del genitivo del persiano antico, distinguendo i pronomi personali dalle restanti forme di genitivo.

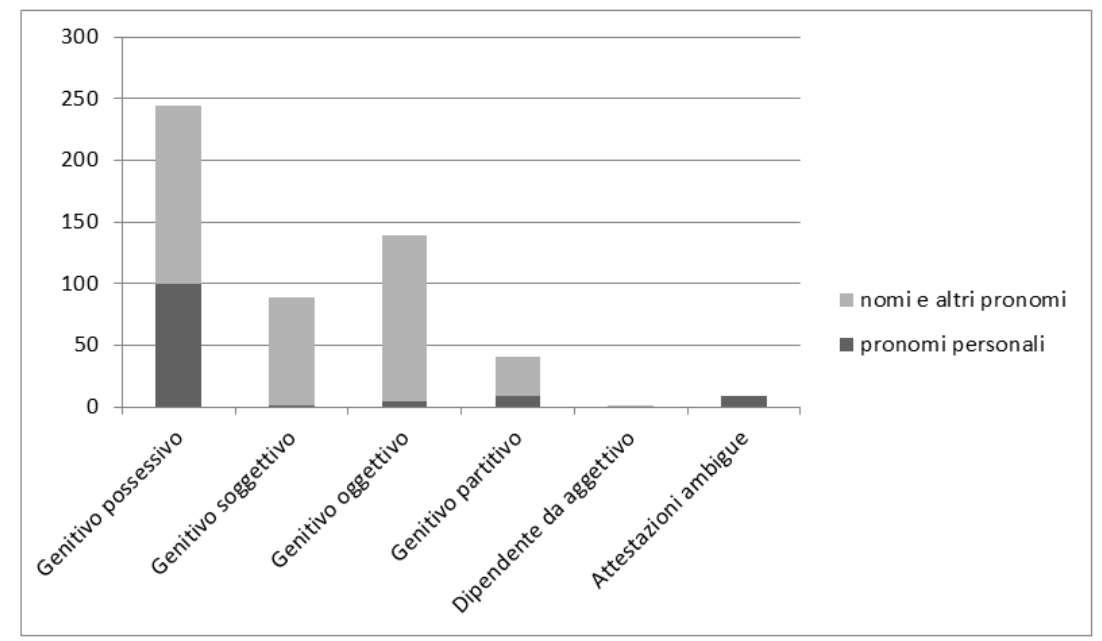

Grafico 2: Attestazioni dei sintagmi genitivali in persiano antico: usi adnominali. 


\subsection{Ruoli e funzioni del genitivo come costituente frasale}

Quando si passino in rassegna le funzioni sintattiche e i ruoli semantici del genitivo a livello di frase, l'avvenuta estensione della gamma funzionale di questo caso nel persiano antico appare più evidente, sia come argomento del verbo sia come aggiunto, vale a dire un elemento extra-nucleare. Dall'analisi dei dati, infatti, emerge che il genitivo è deputato all'espressione di una molteplicità di relazioni grammaticali e ruoli semantici schematizzati infra nella tabella 2 - molti dei quali sono tipici del dativo.

Le costruzioni con un sintagma genitivale in funzione di argomento verbale sono di vari tipi. Più in particolare, esso può costituire il secondo o il terzo argomento di verbi. Nel primo caso, si tratta per lo più di verbi bivalenti provvisti di un basso grado di transitività ${ }^{8}$, ad esempio, pati-x̌say- 'regnare su', in dipendenza dal quale il genitivo codifica un Paziente non prototipico, come nell'attestazione in (1)':

(1) adamšām patiyax̌̌ayai (Schmitt 2000: XPh 16-17) ${ }^{10}$

"I ruled them".

In persiano antico, inoltre, il genitivo può esprimere il ruolo semantico di Agente nella costruzione nota come manā krtam e il Possessore nelle costruzioni esprimenti "possesso" a livello di frase.

Infine, nell'intero corpus è documentata solo un'attestazione, riportata qui di seguito in (2), nella quale un genitivo è usato con un verbo intransitivo di moto: il nome in genitivo costituisce il secondo argomento del predicato e codifica la Meta animata, un ruolo tipico del dativo.

(2) imā dahyāva tayā manā patiyāî̌a (Schmitt 1991: DB 1.13)

"These (are) the countries which fell to my lot"11.

Diverso è il tipo di costruzione con il genitivo come terzo argomento di verbi trivalenti: il genitivo di solito esprime la funzione di oggetto indiretto e codifica il Ricevente (cfr. § 3.) o il Destinatario, ruoli che in una prospettiva comparativa interlinguistica sono entrambi tipici del dativo.

(3) Aāti Dārayavauš xšāyatiya iyam dahyāūš Pārsa tayām manā Auramazdā frābara (Schmitt 2000: DPd 5-8)

"Proclaims Darius, the king: This country Persia, which Auramazdā bestowed upon me".

8 Cfr. Hopper - Thompson 1980.

9 Tra gli altri verbi bivalenti che, a causa del basso grado di transitività della predicazione, prevedono un genitivo come secondo argomento va verosimilmente annoverato $d(u) r u j-$ 'mentire, ingannare', del quale l'oggetto sintattico può essere codificato da un genitivo o da un accusativo (si vedano, tra gli altri, Meillet Benveniste 1931, 210; Pompeo - Benvenuto 2011, 96).

10 Le forme di genitivo di volta in volta prese in esame sono evidenziate dal grassetto. I testi degli esempi in persiano antico e le traduzioni sono tratti dalle edizioni di Schmitt 1991; 2000; 2009.

11 In realtà, l'interpretazione di questo passo è piuttosto controversa. Il problema centrale, come osserva lo Schmitt $(1991,49)$ cui rimandiamo per i dettagli sulla questione, è costituito dal fatto che, nonostante questa sequenza sembri implicare un movimento concreto, non si può escludere a priori la possibilità di un senso figurato. 
Infine, il genitivo argomento del verbo Aand- 'sembrare' codifica l'Esperiente, anche questo un ruolo che, in prospettiva comparativa, risulta tipico del dativo.

Quando si passi ad analizzare le attestazioni del genitivo in funzione di aggiunto, $\mathrm{i}$ dati sono ancora più limitati. A tale proposito, ci limitiamo a osservare che il Beneficiario (esemplificato in 4) è un ruolo semantico che nelle altre lingue indoeuropee è codificato dal cosiddetto dativus commodi:

(4) haya šiyātim adā martiyahyā (Schmitt 2000: XPh 3-4)

"who created blissful happiness for man".

Per concludere questa breve rassegna, può essere utile riassumere in forma tabellare $\mathrm{i}$ dati sinora illustrati. Nella tabella 2, le prime tre colonne elencano i ruoli semantici e lo status sintattico (modificatore di un nome o costituente frasale) del genitivo del persiano antico; la quarta colonna evidenzia come tutti i ruoli codificati dal caso in esame, fatta eccezione per il Partitivo e il Tempo determinato, presentino il tratto semantico [+umano], il quale, a sua volta, implica "animatezza" e, verosimilmente, "controllo"; la colonna più a destra, infine, indica i casi che codificano questi ruoli e queste funzioni tipicamente secondo una prospettiva comparativa interlinguistica.

\begin{tabular}{|c|c|c|c|c|}
\hline RUOLI SEMANTICI & STATUS SINTATTIC & & TRATTI SEMANTICI & \\
\hline & $\begin{array}{l}\text { Modificatore } \\
\text { di nome }\end{array}$ & $\begin{array}{l}\text { Costituente } \\
\text { frasale }\end{array}$ & {$[ \pm$ umano] } & \\
\hline Partitivo & + & + & \pm & \\
\hline Tempo determinato & - & + & - & GENITIVO \\
\hline Possessore & + & + & + & \\
\hline Agente & + & + & + & GENITIVO/ \\
\hline $\begin{array}{l}\text { Paziente } \\
\text { (non prototipico) }\end{array}$ & + & + & + & DATIVO \\
\hline Ricevente & - & + & + & \\
\hline Destinatario & - & + & + & \\
\hline Esperiente & - & + & + & DATIVO \\
\hline Beneficiario & - & + & + & \\
\hline Meta animata & - & + & + & \\
\hline
\end{tabular}

Tab. 2: Ruoli semantici codificati dal genitivo e sue codifiche.

A nostro avviso, la varietà di funzioni e ruoli del genitivo del persiano antico, che di primo acchito sembrano tra loro profondamente disomogenei, può essere spiegata secondo la prospettiva teorica della Cognitive Grammar $^{12}$. In particolare, per quanto concerne il fenomeno qui analizzato, questo tipo di approccio offre chiari vantaggi sia per la descrizione della categoria del genitivo persiano antico sia per la comprensione del processo di sincretismo. In primo luogo, infatti, ci consente di chiarire lo status relativo di

12 Per un contributo recente sull'analisi dei casi secondo questo tipo di approccio teorico, si veda Luraghi 2009 e i riferimenti bibliografici ivi contenuti. 
ciascuna delle funzioni del genitivo e di strutturare e organizzare le relazioni tra i vari tipi di uso documentati in un quadro coerente, che si configura in modo ottimale come una categoria radiale. Secondo quanto osservato da $\mathrm{S}$. Luraghi ${ }^{13}$, una categoria radiale è un tipo di categoria prototipica, internamente strutturata e caratterizzata dalla presenza di un membro centrale (il prototipo) provvisto di tutti i tratti rilevanti della categoria medesima ${ }^{14}$. Oltre al prototipo, appartengono alla categoria altri membri più periferici, i quali sono estensioni del prototipo - prevalentemente per mezzo di processi di tipo metaforico rispetto al quale possiedono i tratti tipici della categoria cui appartengono in minor grado: tanto minore sarà la quantità di tratti presenti in un dato membro della categoria, tanto più questo sarà periferico e lontano dal prototipo. Da ciò deriva l'etichetta di "categoria radiale" usata per definire questo tipo di strutturazione concettuale. Di conseguenza, quando tale modello interpretativo è applicato a un caso polisemico, vale a dire caratterizzato da una gamma di usi differenti, questi vengono a essere organizzati secondo una struttura radiale, dove la funzione centrale - solitamente la più frequente non solo nella lingua in esame ma anche in prospettiva tipologica - costituisce il prototipo dal quale si dipartono le altre funzioni, relativamente meno frequenti e più periferiche.

In secondo luogo, i modelli di rappresentazione della polisemia, stabiliti empiricamente in base a dati di tipo sincronico, consentono predizioni riguardanti modelli di polisemia possibili o impossibili anche in una prospettiva diacronica, contribuendo in tal modo alla ricostruzione di un dato processo di sincretismo. A tale proposito, appare particolarmente interessante quanto osservato da $\mathrm{K}$. Nikiforidou ${ }^{15}$. La studiosa greca, infatti, nota che, data una gamma di funzioni e ruoli codificata da un caso in una specifica fase linguistica, gli usi centrali in prospettiva sincronica, vale a dire prototipici, mostrano anche una tendenza a essere quelli diacronicamente più persistenti, resistendo così anche a processi di sincretismo.

Orbene, se rappresentiamo i dati quantitativi illustrati sopra (cfr. grafici 1-2) con uno schema che raffigura il genitivo del persiano antico come una categoria radiale (fig. 1), ne risulta una categoria internamente strutturata dove il ruolo del Possessore, che è il più produttivo, costituisce il membro centrale, mentre gli altri ruoli vengono interpretati come estensioni metaforiche del Possessore: tanto più i differenti ruoli sono distanti dal centro/prototipo, tanto più essi sono periferici e meno produttivi $(\S 4.2 .)^{16}$.

\section{DALL'INDOEUROPEO AL PERSIANO ANTICO}

L'analisi delle funzioni del genitivo fin qui condotta fornisce le basi necessarie per inquadrare la discussione sulla natura del sincretismo. Secondo la spiegazione più classica di tale fenomeno, la fusione di morfemi di casi o l'eventuale perdita di alcuni di essi è motivata da mutamenti fonetici che causano l'erosione degli esponenti morfologici.

13 Luraghi 2009, 139.

14 Per la definizione di "categoria radiale", S. Luraghi si basa sul noto lavoro di Lakoff 1987.

15 Nikiforidou 1991.

16 G. Haig (2008, cap. 2) propone un'ipotesi differente e teorizza che il genitivo persiano antico sia deputato alla codifica dell'ampia categoria della "Partecipazione indiretta", nell'ambito della quale il ruolo nucleare sarebbe costituito dal Beneficiario; da quest'ultimo deriverebbero tutte le altre funzioni attestate per il caso in esame. 
Il materiale offerto dal persiano antico impone una certa cautela, dal momento che la nostra comprensione del sistema morfologico è condizionata dalle varie lacune (soprattutto per quanto riguarda il plurale) a causa del corpus limitato. Inoltre, l'esatta definizione delle desinenze nominali è talvolta compromessa dalla complessa natura del sistema di scrittura cuneiforme e dalle convenzioni ortografiche che possono in certa misura celare alcuni aspetti significativi della morfologia ${ }^{17}$.

In prospettiva storico-comparativa è interessante notare che la marca casuale del genitivo del persiano antico risale direttamente al genitivo proto-indoeuropeo, sia nella classe dei tematici $\left(-a h y \bar{a}<\right.$ ie. $*_{-}$esyo/*-osyo $)$che degli atematici $\left(-a<\right.$ ie. $\left.{ }^{*} e s / *_{-o s} / *_{-s}\right)$, mentre l'originaria terminazione del dativo non ha lasciato riflessi diretti negli esponenti morfologici attestati e sembra essere andata completamente perduta.

Peraltro è difficilmente pensabile che siano stati i processi fonetici le forze trainanti del sincretismo. Infatti, secondo le leggi fonetiche operanti in persiano antico, gli esiti dell'originario dativo indoeuropeo si sarebbero dovuti conservare. Nel singolare, il riflesso delle desinenze originarie del dativo indoeuropeo - *-oi e $*_{-}$ei rispettivamente della classe dei tematici e degli atematici - sarebbe dovuto essere il dittongo -ai che in persiano antico è ben attestato in fine di parola. Si veda, ad esempio, Parsai 'in Persia' $\approx$ gr. oǐkor 'a casa' o yadatai 'egli adora' $\approx$ gr. $\alpha$ ¿ $\varepsilon \tau \alpha$ l. Per quanto concerne le terminazioni del plurale, gli esiti dello strumentale, dativo e ablativo indoeuropei, tutti caratterizzati da *-bh- iniziale, sono attestati nella forma del persiano antico di ablativo/strumentale (cfr. bagaibišs). Ciò nonostante, in questa lingua anche nel plurale le funzioni del dativo sono codificate dalla desinenza del genitivo.

Infine, nelle lingue indoeuropee è possibile osservare che quando il sincretismo è dovuto a sviluppi fonetici certi, il morfema sincretico conserva traccia, nelle varie classi flessive, di tutte le terminazioni pre-sincretiche che si sono fuse in esso $^{18}$. In persiano antico, invece, le forme di genitivo non mantengono alcuna delle originarie desinenze dativali in nessuno dei paradigmi. Di conseguenza, possiamo concludere che in persiano antico il sincretismo, verosimilmente, non è il risultato di mutamenti fonetici. Le cause della perdita di uno dei casi, vale a dire del dativo, e della conseguente riassegnazione delle sue funzioni al genitivo vanno, quindi, ricercate altrove.

\section{MOTIVAZIONI SINTATTICHE E SEMANTICHE DEL SINCRETISMO}

Il costituirsi del caso genitivo del persiano antico, che abbiamo visto essere indipendente dal sincretismo formale, deve essere imputato, quindi, a cambiamenti nell'area funzionale dei casi coinvolti. Tale fusione funzionale, a nostro avviso, corrisponde in modo significativo al tipo di sincretismo ottenuto attraverso ciò che Meiser ${ }^{19}$ definisce

17 Cfr. Schmitt 2004, 721. Per una presentazione dettagliata del sistema cuneiforme del persiano antico e sulle convenzioni ortografiche che lo caratterizzano si rimanda a D'Erme 1983; 1990; Mancini 1992 e al recente lavoro di Rossi 2005.

18 Cfr. Meiser 1992.

19 Meiser (1992) distingue due tipi di sincretismo funzionale: shift of extensions e congruence of extensions. Nel primo, l'ampliamento dell'estensione di una categoria a scapito di un'altra fa sì che quest'ultima sia attestata dapprima in uno stato relittuale, per poi, anche se non sempre, scomparire del tutto. Al contrario, nel secondo tipo, due terminazioni tendono a convergere nell'espressione dello stesso dominio semantico. La differenza 
shift of extensions ovvero dalla sovraestensione funzionale (semantica e sintattica) di una categoria in concomitanza con la riduzione del campo di applicazione e conseguente scomparsa di un'altra. In tale processo, quando un morfema inizia a ricoprire lo spazio semantico precedentemente occupato da un altro e due diverse espressioni entrano in competizione nella codifica dello stesso contenuto, la soppressione della categoria grammaticale in un certo qual modo sentita come ridondante è il mezzo attraverso il quale il sistema risolve lo squilibrio nel rapporto tra espressione e contenuto creato dalla sinonimia.

Date tali premesse, al fine di comprendere quanto più possibile il processo di sovraestensione funzionale del genitivo è opportuno considerare gli aspetti sintattici e semantici che possono averlo favorito.

\subsection{Aspetti sintattici}

Per quanto riguarda il livello sintattico, in base ai dati offerti dalla comparazione con l'avestico e l'indiano, la sovraestensione funzionale sembra essere stata causata da una parziale sinonimia della struttura argomentale delle costruzioni nelle quali i due casi, genitivo e dativo, occorrono ${ }^{20}$.

Innanzitutto, sia genitivo che dativo sono usati tanto in avestico che in vedico per la codifica dell'oggetto non prototipico in dipendenza da verbi bivalenti. Il genitivo è usato con verbi che significano 'regnare, avere potere su' o con verbi di percezione/comprensione, mentre il dativo è associato a verbi di lode, preghiera, propiziazione o che significano 'venire in aiuto di'. In tutti questi contesti, entrambi i casi indicano che il secondo argomento del verbo non è un Paziente prototipico e che, avendo un basso grado di coinvolgimento, non subisce un cambiamento di stato. In altre parole, i due casi codificano il Paziente di verbi caratterizzati da bassa transitività ${ }^{21}$.

Tuttavia, la principale area di congruenza dell'estensione funzionale si verifica nell'espressione del possesso predicativo. In indoiranico l'unica strategia sintattica usata per esprimere l'esistenza di una relazione possessiva prevede che la cosa posseduta sia codificata come il soggetto grammaticale di un verbo copulare/esistenziale (iranico antico $a h$ - 'essere' o bav- 'essere, divenire'), mentre il Possessore è espresso per mezzo del genitivo o del dativo ${ }^{22}$. In avestico, il dativo in combinazione con ah- 'essere' o bav'essere, divenire' indica la proprietà o il possesso a partire dal significato di 'esserci per, essere destinato a' (vedi esempio 5$)^{23}$.

tra i due tipi di sincretismo appare nella significativa diversità dei rispettivi esiti: sul piano morfologico, il sincretismo mediante sovraestensione porta inevitabilmente a una perdita completa di uno dei due morfemi, mentre il sincretismo attraverso sovrapposizione delle estensioni dà luogo alla rifunzionalizzazione delle desinenze originarie (i morfemi delle categorie precedentemente distinte diventano allomorfi della stessa categoria) e alla loro ridistribuzione morfologica all'interno del paradigma.

20 Sul sincretismo motivato «by the existence of synonymous argument structure constructions» si rimanda a Barðdal - Kulikov 2009.

21 Per i parametri della transitività e la variazione nella codifica grammaticale dei partecipanti all'evento descritto dal verbo si rimanda a Hopper - Thompson 1980. Per uno studio dettagliato sugli aspetti semantici e pragmatici della codifica degli oggetti non prototipici in vedico e indoiranico si veda anche Dahl 2009; in stampa.

22 Cfr. Èdel'man 1975; Bauer 2000; Viti 2004

23 Reichelt 1909, 253; Edel'man 1975, 152. 
(5) ušta buiiāt ahmāi naire (Y. 62.1)

"Salvation be to this man".

Oltre che con il dativo, i verbi $a h$ - e $b a v$-ammettono una costruzione con il genitivo per esprimere in particolare 'appartenere a' (esempio 6).

(6) ciš ahī kahiiā ahī (Y. 43.7)

"Who are you? Whose are you?".

La stessa situazione può essere osservata nei testi vedici ${ }^{24}$, come documentato negli esempi 7 e 8 .

(7) asmä́kam astu kévalah $(R V 1.7 .10)$

"he will belong to us completely";

(8) gambhīré cid bhavati gādhám asmai (RV 6.24.8)

"even in deep water he has a ford".

Infine, in molte lingue indoeuropee, genitivo e dativo possono essere usati per codificare il ruolo dell'Agente ${ }^{25}$ con forme verbali sia di modo finito che non finito (esempi 9 e 10). Per quanto riguarda le forme verbali non finite, vale la pena notare che l'Agente animato in dipendenza dai participi in -ta- può essere espresso tanto in indiano antico che in iranico antico sia dal genitivo (cfr. 9a-10a) sia, più raramente, dal dativo (cfr. 9b-10b).

GENITIVO

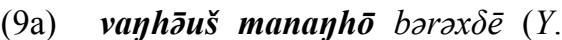

48. 6)

"dont les mérites sont reconnus par la divine Pensée"26

(10a) kád ù nú asya ákrtam (RV 8.66.9)

"von him ungetan bzw. nicht zu vollbringen" 27

In conclusione, gli esempi illustrati in precedenza avvalorano l'ipotesi che le aree funzionali di genitivo e dativo in indoiranico fossero in parte sinonimiche. Tale parziale sovrapposizione di funzioni sintattiche può aver costituito uno dei fattori che, a causa della tendenza delle lingue a evitare la ridondanza di forme grammaticali, ha provocato la fusione di costruzioni con struttura argomentale uguale o simile ${ }^{28}$, con conseguente perdita delle distinzioni casuali.

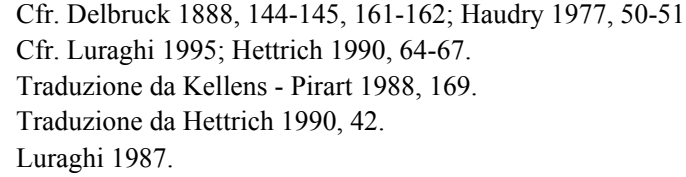


La direzione di questo cambiamento è confermata, inoltre, dalla deriva osservabile in indoiranico nell'evoluzione di entrambi i casi: il dativo va estinguendosi progressivamente in avestico recente e indiano antico, ed è ormai un relitto in medio-indiano ${ }^{29}$, mentre il genitivo va estendendosi a un numero crescente di settori che sfuggono alla sua area semantica originaria, sostituendo il dativo in molti contesti.

Per quanto riguarda l'avestico, è sufficiente qui ricordare ciò che era stato già osservato dal Reichelt ${ }^{30}$ riguardo al dativische Genitiv ${ }^{31}$ : l'alta frequenza del genitivo adnominale ha determinato la progressiva estensione di questo caso a scapito della sfera di azione del dativo interessando in una prima fase (avestico gāthico) gli usi adnominali del dativo e solo successivamente le funzioni del dativo a livello di frase, soprattutto nell'espressione del Beneficiario. Successivamente, in avestico recente, il fenomeno si sarebbe spinto fino a comprendere la funzione sintattica di oggetto indiretto e il ruolo del Destinatario. Tuttavia, nonostante questa espansione progressiva del genitivo a spese del dativo, il primo non riesce a prevalere definitivamente sul secondo.

\subsection{Aspetti semantici e cognitivi}

Esaminiamo ora gli aspetti semantici e cognitivi che possono essere considerati soggiacenti alla sovrapposizione funzionale tra il genitivo, caso che ha come ruolo nucleare il Possessore, e il dativo, caso nella cui gamma funzionale il Ricevente costituisce un ruolo di fondamentale importanza (cfr. tabb. 1-2) ${ }^{32}$. Si tratta, in particolare, di individuare quali sono i ruoli primariamente coinvolti nel processo d'intersezione e le modalità di quest'ultimo.

Per quanto concerne le relazioni tra il Possessore e i ruoli di Esperiente, Agente, Paziente e Partitivo, rimandiamo al lavoro fondamentale di K. Nikiforidou ${ }^{33}$, la quale teorizza che tutti questi ruoli sono collegati in maniera diretta al Possessore per mezzo di differenti estensioni metaforiche. D'altro canto, per quanto riguarda i ruoli di Ricevente, Destinatario e Beneficiario - tutti assenti dai Metaphorical mappings della Nikiforidou - i quali in prospettiva interlinguistica sono espressi dal dativo nonché tipici di questo caso, abbiamo proposto ${ }^{34}$ che essi sono collegati al significato centrale del Possessore per mezzo di estensioni metaforiche, costruite su uno schema sostanzialmente analogo. A nostro avviso, infatti, queste estensioni possono essere schematizzate nel modo seguente: 1) il Ricevente è concepito come Possessore in prospettiva di qualcosa che è trasferito da una persona ad un'altra; 2) il Destinatario è concettualizzato come Possessore in prospettiva di un'informazione detta o, comunque, trasmessa; 3) il Beneficiario è concepito come Possessore in prospettiva degli effetti positivi o negativi di una data azione. A proposito di quest'ultimo processo, è opportuno notare che nella letteratura sull'argomento sono

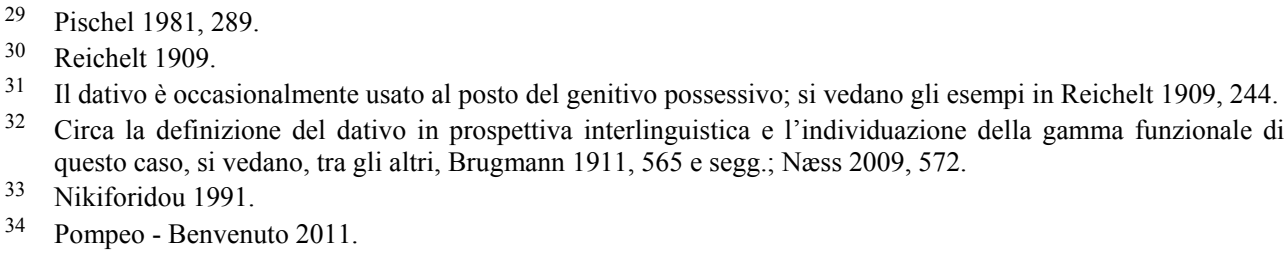


individuati differenti tipi di Beneficiario. Tra questi va menzionato il "Recipientbeneficiary" ${ }^{35}$ che si caratterizza come il tipo di Beneficiario più simile al ruolo di Possessore da un punto di vista semantico e, talora, anche formale. Questa etichetta è usata, infatti, per designare chi trae beneficio da una data azione grazie alla quale diventa anche il Ricevente e, verosimilmente, il Possessore di qualcosa. Ne è un tipico esempio la frase inglese The dentists baked the phonetician a cake, dove il fonetista ottiene un duplice vantaggio dall'azione del dentista, poiché non solo non cuoce di persona la torta ma ne è anche il Ricevente e Possessore ${ }^{36}$. Il Beneficiario codificato dal genitivo persiano antico sembra corrispondere proprio a quest'ultimo tipo.

Lo schema che proponiamo per rappresentare il processo metaforico soggiacente ai tre tipi di estensione illustrati è riportato nella figura 2.

Purtroppo non è possibile stabilire una gerarchia cronologica tra le tre estensioni metaforiche suddette, poiché la scarsità dei dati testuali non consente di condurre per il persiano antico un'indagine di tipo diacronico, in quanto, come già osservato, nelle iscrizioni achemenidi il dativo è completamente scomparso. L'unico elemento che risulta in modo evidente dalla nostra analisi è che tutti e tre i ruoli (Ricevente, Destinatario e Beneficiario) sono strettamente legati al Possessore.

Anche i dati provenienti da studi di stampo tipologico confermano questa ipotesi. Infatti, l'esistenza di un'unica forma deputata alle funzioni altrove tipiche rispettivamente di genitivo e dativo è osservabile in varie lingue, in particolare in quelle australiane e austronesiane ${ }^{37}$. Lo stesso tipo di fenomeno, inoltre, può coinvolgere non i casi nella loro intera gamma funzionale, bensì solo alcuni dei ruoli semantici qui considerati (in particolare, Possessore, Ricevente e Beneficiario) secondo vari modelli di polisemia, come già accennato a proposito del Recipient-beneficiary. La polisemia di questo secondo tipo è diffusa in molte lingue appartenenti a famiglie differenti, e non di rado è documentata anche in sistemi linguistici dove coesistono dativo e genitivo. Basti pensare al costrutto noto come "Possessore esterno" o alle costruzioni del possesso predicativo con il verbo 'essere' che, in molte lingue indoeuropee di antica attestazione, prevedono l'uso del dativo e del genitivo rispettivamente per esprimere "possesso" o "appartenenza", documentando l'esistenza di una forte connessione semantica tra Possessore e Ricevente o Beneficiario ( 4.1. $)^{38}$.

Resta un ultimo aspetto da considerare. Dall'analisi condotta nei paragrafi precedenti è emerso che gli unici ruoli semantici comparativamente tipici del dativo (cfr. tab. 1) a non essere codificati dal genitivo del persiano antico sono la Meta e lo Scopo. In effetti, come osservato nel paragrafo 2.2., nell'intero corpus è documentata un'unica attestazione di sintagma genitivale esprimente la Meta animata, in un passo dall'interpretazione abbastanza problematica. A tale proposito, secondo un approccio di tipo cognitivo, il fatto che il genitivo semplice, che ha assunto le funzioni del dativo, non esprima la Meta e lo Scopo trova una possibile spiegazione nella mancanza di una connessione diretta a livello

35 Cfr. Van Valin - La Polla 1997, 383 e segg.

36 Kittilä - Zúñiga (eds.) 2010, 253.

37 Cfr., fra gli altri, Lander 2009 e Næss 2009.

38 Si veda, ad esempio, il lavoro di Kittilä - Zúñiga (eds.) 2010. 
semantico-cognitivo tra il Possessore e questi due ruoli. Tale situazione è schematizzata nella mappa semantica tratta da $\operatorname{Malchukov}^{39}$ (fig. 3), elaborata sulla base di dati tipologici ${ }^{40}$. Nondimeno, a causa della scarsità del materiale documentario, la nostra proposta, pur se fondata su dati semantico-cognitivi e tipologici, resta una mera ipotesi.

Per riassumere quanto illustrato in questo paragrafo, la contiguità semantica e cognitiva tra Ricevente/Destinatario/Beneficiario e Possessore, fondata sull'interpretazione dei primi tre ruoli come Possessori in prospettiva, può aver determinato uno spostamento nell'estensione funzionale di genitivo e dativo che ha condotto al sincretismo dei due casi precedentemente distinti.

\section{CONSIDERAZIONI CONCLUSIVE}

I risultati della nostra analisi suggeriscono che il caso genitivo del persiano antico debba essere interpretato come una categoria polisemica nell'ambito della quale l'espressione del Possessore costituisce la funzione centrale, mentre le altre funzioni attestate, tipiche o meno del genitivo, vanno interpretate come estensioni radiali e metaforiche del significato nucleare. La centralità della funzione possessiva in persiano antico è di particolare rilievo anche in conseguenza del fatto che questa lingua non possiede né pronomi possessivi né un verbo transitivo che significhi "possedere, avere".

Lo studio del materiale documentario del persiano antico, inoltre, induce a ipotizzare che la scomparsa del dativo proto-iranico non sia stata provocata da mutamenti fonetici, ma, piuttosto, sia stata determinata dall'estensione funzionale del genitivo alle spese del dativo. Appare verosimile che lo spostamento di estensione sia stato provocato sia dalla fusione delle costruzioni sintattiche con analoga struttura argomentale che dalla contiguità semantica dei ruoli codificati dai due casi interessati dal processo sincretico, che ha coinvolto, in particolare, Possessore, Ricevente, Destinatario e Beneficiario.

Infine, i dati derivanti dalla comparazione interlinguistica genealogica e tipologica sembrano avvalorare l'ipotesi qui proposta.

\section{BIBLIOGRAFIA}

BARĐDAL, J. - KULIKOV, L.I.

2009 Case in decline: A. MalchuKov - A. Spencer (eds.), The Oxford handbook of case, Oxford 2009, pp. 470-478.

BAUER, B.

2000 Archaic syntax in Indo-European. The spread of transitivity in Latin and French, Berlin 2000.

BLAKE, B.J.

$2001 \quad$ Case, Cambridge 2001².

39 Malchukov 2010

40 Secondo la definizione di Haspelmath $(2003,213)$ «a semantic map is a geometrical representation of functions in 'conceptual/semantic space' that are linked by connecting lines and thus constitute a network. The configuration of functions shown by the map is claimed to be universal.». 
BRUGMANN, K.

1911 Grundriss der vergleichenden Grammatik der indogermanischen Sprachen, 2, 2, Strassburg 1911.

DAHL, E.

2009 Some Semantic and Pragmatic Aspects of Object Alternation in Early Vedic: J. BARĐDAL - S. Chelliah (eds.), The Role of Semantic, Pragmatic and Discourse Factors in the Development of Case, Amsterdam 2009, pp. 23-55.

in stampa The Partitive Genitive in Indo-Iranian: S. LuRAGHI - T. Huumo (eds.), Papers from the D'ERME, G. workshop on partitives at the SLE 43rd Annual Meeting, Berlin in stampa.

1983 Aspetti grafici e fonetici della scrittura antico-persiana: AIQN 43 (1983), pp. 429-477.

1990 Elamico e antico-persiano; affinità stilistiche tra i due sistemi scrittorii: G. GNOLI - A PANAINO (eds.), Proceedings of the First European Conference of Iranian Studies (Turin, September 7th-11th, 1987), Roma 1990, pp. 69-83.

DELBRÜCK, B

$1888 \quad$ Altindische Syntax, Darmstadt 1888.

ÈDEL'MAN, D.I.

1975 Les verbes 'être' et 'avoir' dans les langues iraniennes: F. BADER (ed.), Mélanges linguistiques offerts à Émile Benveniste, Louvain 1975, pp. 151-158.

HAIG, G.

2008 Alignment change in Iranian languages. A construction grammar approach, Berlin - New York 2008.

HASPELMATH, M.

2003 The geometry of grammatical meaning. Semantic maps and cross-linguistic comparison: M. Tomasello (ed.), The new psychology of language, vol. 2, Mahwah 2003, pp. 211242.

HAUDRY, J.

1977 L'emploi des cas en védique: introduction à l'étude des cas en Indo-Européen, Lyon 1977.

HeTtRich, H.

1990 Der Agens in passivischen Sätzen altindogermanischer Sprachen, Göttingen 1990.

HOPPER, P. - THOMPSON, S.

1980 Transitivity in Grammar and Discourse: Language 56.4 (1980), pp. 251-299.

KELLENS, J. - PiRART, E.

$1988 \quad$ Les textes vieil-avestiques, vol. I, Wiesbaden 1988.

KITTILÄ, S. - ZÚÑIGA, F. (eds.)

2010 Benefactives and Malefactives. Typological perspectives and case studies, Amsterdam Philadelphia 2010.

LAKOFF, G.

1987 Women, fire, and dangerous things, Chicago 1987.

LANDER, Y.

2009 Varieties of Genitive: A.L. MALCHUKOV - A. SPENCER (eds.), The Oxford handbook of case, Oxford 2009, pp. 581-592.

LURAGHI, S.

1987 Patterns of case syncretism in Indo-European languages: A. GiaCALONE RAMAT - O. CARruba - G. Bernini (eds.), Papers from the 7th International Conference on Historical Linguistics, Amsterdam - Philadelphia 1987, pp. 355-371.

1995 Prototypicality and agenthood in Indo-European: H. ANDERSEN (ed.), Historical linguistics, Amsterdam 1995, pp. 254-268. 
2000 Synkretismus: G. Boolj - C. LeHMann - J. Mugdan (eds.), Morphology. An international handbook on inflection and word-formation, Berlin - New York 2000, pp. 638-647.

2009 Case in Cognitive Grammar: A.L. MalchuKov - A. SPEncer (eds.), The Oxford handbook of case, Oxford 2009, pp. 136-150.

MALCHUKOV, A. L.

2010 Analyzing semantic maps: a multifactorial approach: Linguistic Discovery 8.1 (2010), pp. 176-198.

MANCINI, M.

1992 Sul sillabismo finale nel cuneiforme achemenide, Viterbo 1992.

Meillet, A. - Benveniste, É.

$1931 \quad$ Grammaire du vieux-perse, Paris 1931

MEISER, G.

1992 Syncretism in Indo-European languages - Motives, process and results: Transactions of NÆSS, Å. the Philological Society 90.2 (1992), pp. 187-218.

2009 Varieties of Dative: A.L. MALCHUKov - A. SPEnCER (eds.), The Oxford handbook of case, Oxford 2009, pp. 572-580.

NIKIFORIDOU, K.

1991 The meanings of the genitive. A case study in semantic structure and semantic change: Cognitive Linguistics 2.2 (1991), pp. 149-205.

PISCHEL, R.

1981 A Grammar of the Prakrit Languages, Delhi 1981.

Pompeo, F. - Benvenuto, M.C.

2011 Il genitivo in persiano antico. Un caso esemplare di categoria polisemica: Studi e saggi linguistici 49 (2011), pp. 75-123.

REICHELT, H.

1909 Avestisches Elementarbuch, Heidelberg 1909.

Rossi, A.V.

2005 La scrittura antico-persiana e la scrittura elamico-achemenide: M. BERNARDINI - N.L. TORNESELlo (a cura di), Scritti in onore di Giovanni M. D'Erme, Napoli 2005, pp. 927942.

SCHMITT, R.

1991 The Bisitun Inscriptions of Darius the Great: Old Persian text (Corpus Inscriptionum Iranicarum, Part I, Inscriptions of Ancient Iran, I: The Old Persian Inscriptions, Texts I), London 1991.

2000 The Old Persian inscriptions of Naqsh-i Rustam and Persepolis (Corpus Inscriptionum Iranicarum, Part I, Inscriptions of Ancient Iran, I: The Old Persian Inscriptions, Texts II), London 2000.

2004 Old Persian: R.D. Woodard (ed.), The Cambridge Encyclopedia of the World's Ancient Languages, Cambridge 2004, pp. 717-741.

2009 Die altpersischen Inschriften der Achaimeniden. Editio minor mit deutscher Übersetzung, Wiesbaden 2009

VAN VAlin, R. D. JR. - LA POLla, R.

1997 Syntax. Structure, meaning and function, Cambridge 1997.

VITI, C.

2004 Funzioni semantiche e pragmatiche nelle strategie di possesso dell'antico indiano: Archivio Glottologico Italiano 89.1 (2004), pp. 41-83. 


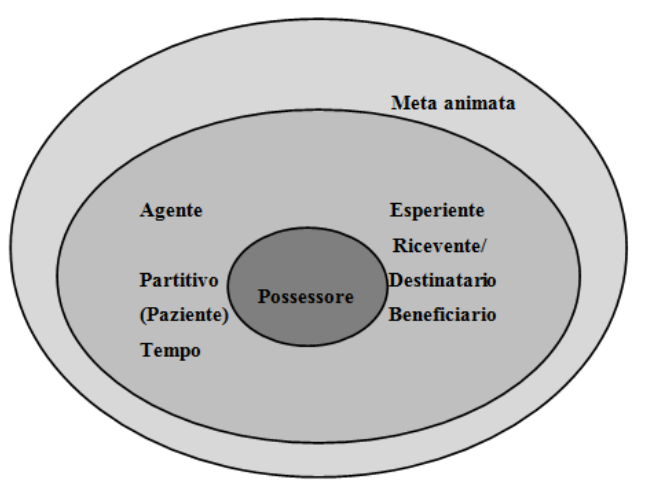

Fig. 1: Il genitivo del persiano antico come categoria radiale.

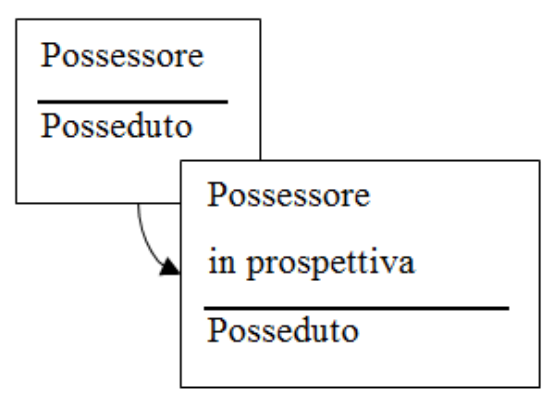

Fig. 2: Schema del processo di estensione metaforica.

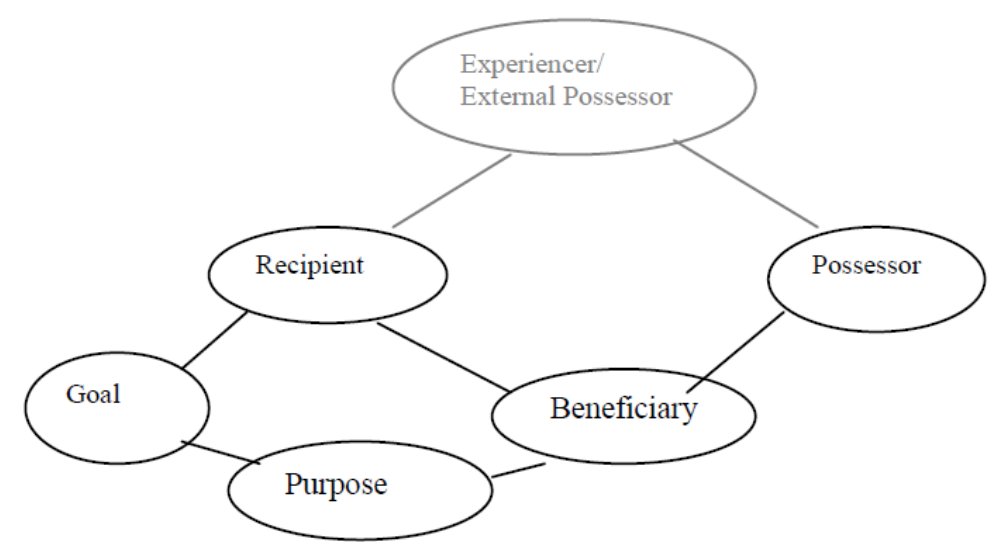

Fig. 3: Possessive-benefactive connection (da Malchukov 2010, 190). 